\title{
A Feature Vector Compression Approach for Face Recognition using Convolution and DWT
}

\author{
Ganapathi V Sagar ${ }^{1}$, Savita Y Barker ${ }^{2}, \mathrm{~K}$ B Raja ${ }^{3}$, K Suresh Babu ${ }^{3}$ and Venagopal K R ${ }^{3}$ \\ ${ }^{1}$ Dr.Ambedkar Institue of Technology,Bangaluru,ganapathi_sagar@yahoo.co.in \\ ²Sai Tektronix Pvt Ltd,Bangaluru,savitha.b@saitecgroup.com \\ ${ }^{3}$ University Visvesvaraya College of Engineering,BangaluruUniversity,Bangaluru
}

\begin{abstract}
The biometric identification of a person using face trait is more efficient compared to other traits as the co-operation of a person is not required. In this paper, we propose a feature vector compression approach for face recognition using convolution and DWT.The one level DWT is applied on face images and considered only LL band. The normalized technique is applied on LL sub band to reduce high value coefficients into lower range of values ranging between Zero and one. The novel concept of linear convolution is applied on original image and LL band matrix to enhance quality of face images to obtain unique features. The Gaussian filter is applied on the output of convolution block to reduce high frequency components to generate fine-tuned feature vectors. The numbers of feature vectors of many samples of single person are converted into a single vector which reduces number of features of each person. The Euclidean distance is used to compare test image features with features of database persons to compute performance parameters. It is observed that the performance recognition rate is high compared to existing techniques.
\end{abstract}

\section{Keywords}

Biometrics; face recognition; DWT; convolution; vector compression

\section{Council for Innovative Research}

Peer Review Research Publishing System

\section{Journal: INTERNATIONAL JOURNAL OF COMPUTERS \& TECHNOLOGY}

Vol. 15 , No. 1

www.ijctonline.com, editorijctonline@gmail.com 


\section{Organization:}

This paper is organized as follows: Section 1 gives brief introduction to Biometrics. Section 2 briefly reviews the literature, Section 3 introduces the new face recognition system based on convolution and compression of database vector features, section 4 describes proposed algorithm, section 5 presents the performance results and compares proposed method with existing methods. Section 6 concludes our work with future directions.

\section{INTRODUCTION}

The physiological and behavioral characteristics of a person are used to recognize a living person which leads to Biometric system. The physiological traits are almost constant throughout the life time of a person and are fingerprint, palm print, Iris, DNA etc. The behavioral traits are varying based on mood, circumstances and environment around a person and examples are voice, signature, gait, keystroke etc. The biometric systems are broadly classified into verification system and identification system. In verifications system, the person's identity is declared by comparing features of a person with earlier stored features in the system which is one to one matching, i.e., the claim is accepted or rejected based on preconditions. In identification system, the person's identity is declared by comparing features of a person with earlier stored features of many persons i.e., one to many matching. The identification system is computationally expensive and complex, whereas, the verification system is simple, less expensive and complex. The general biometric system has enrollment section, test section and matching sections. The biometric traits of persons are captured using sensors. The captured images are preprocessed and features are extracted to create database in the enrollment section. The biometric trait to be tested is captured using sensor, preprocessed and features are extracted to create single feature vector or matrix in the test section. The matching section is used to compare test biometric trait features with database biometric trait features to identify a person based on distance formulae or classifiers. The advantages of biometric identification system compared to traditional methods such as personnel identification number (PIN), identity card, smart card etc., are the biometric traits can't be lost as traits are attached to a person. The face recognition is used in online image search, surveillance in crowd, identification of terrorist in a mob, entry into corporate offices etc. The challenges in face recognition are variations in the light intensity, pose variations, hair occlusions, blinked eye etc.

Novel aspects of this proposed method are:

The LL-band coefficients are normalized to convert high coefficient values between Zero and one.

The unique features are generated by convolving original face image with normalized LL-band matrix.

The Gaussian filter is applied on convolution output to reduce high frequency noise components.

Many feature vectors of single person are converted into single unique feature vector by compression to reduce more number of samples per person to one sample person.

\section{LITERATURE REVIEW:}

In this section, the existing techniques of face recognition are discussed.

Ahilandeswari et al., [1] proposed multimodal biometric system based on facial, fingerprint and speech. The Eigen faces are used for face identification, minutia features are used for fingerprint matching and cepstral analysis is used for speech identification. The features of all three traits are fused at matching level to enhance performance of architecture.Jianwn wan et al., [2] proposed a novel cost sensitive semi supervised discriminate analysis called PCSDA. Using a simple L2 approach to predict the label of unlabeled data and then learns the projection direction by incorporating costs into both labeled data andunlabeled data comparing wits CS3DA, PCCDA approaches. Using L2 approach to predict the label of unlabeled face images is more accurate and robust than the spares representation. Using CS3DA method approximates the pair wise Bayesian risk only when the classes are balanced and without outliers in data base sets. Hailing Zhou et al., [3] proposed recent advances on face recognition. 3-D data includes facial geometry information, increasing robustness to viewpoints and elimination variation compared with usual images. 3-D face recognition can achieve better recognition accuracy then the usual face recognition, although 3-D data are insensitive to illumination variations, it is still difficult to recognize faces in the options of visible light. Multimodal modalities can achieve better performance than a single modality. IR images are acquired for 2-D imaging sensors, IR face recognition also suffer from sensibility to pose variations. 
Rupali L Telgad et al., [4] proposed biometric system based on facial and fingerprint. The Euclidean distance matches are used for face and fingerprint identification. Minutiae and Gabor filter features are used for fingerprint recognition. Principal component Analysis (PCA)is used for face identification and dimensionality reduction. The feature of two traits approach provides a good result. The recognition rate is increased and the error rate is decreased with the help of two traits. Madeena Sultana et al., [5] proposed illumination insensitiveness of the sub bands of Dual-Tree Complex Wavelet Transform (DTCWT) based on different scales. Extensive illumination variations produces high recognition rate even with a single sample using novel face recognition system approach and weighted fusion of low and high frequency sub bands are used for feature extraction. To identify the adoptive weights during uncertain illumination conditions, a novel fussy weighting scheme are used and adaptive normalization approach is applied for illumination quality announcement of the poor lit samples while retaining the good quality samples. Jiwen Lu et al., [6] proposed multiple statistic features and localized multi-feature metric learning method for a new image set based face recognition identification. Two kernel based metric learning algorithms called localized multi-kernel metric learning and localized multi-kernel multi-metric learning are used for extracting effectively combined multiple statistic features from face image set. Efficient kernel approximation methods are used to improve the kernel estimation speed for specific combined statistic features.

original LBP pattern. The performance of CLBP features are classified using Support Vector Machine classifier.XuXiaona et al., [8] Proposed a kernel based feature fusion algorithm is applied on multimodal recognition system based on ear and face. The kernel principal component analysis (KPCA) algorithm is used for multimodal recognition based on ear and profile face performs better than ear or profile face for unimodal biometric identification. Sushama $S$ patil et al., [9] proposed fingerprint image enhancement technique $\mathbf{S}$, Basically there are two types of fingerprint identification system, automatic fingerprint Authentication system and automatic finger print verification/identification system are uses for recent advances in fingerprint image enhancement to change in finger position, finger condition and finger pressure.

SheetalChaudhary et al., [10] Proposed a multimodal biometric recognition system based on fusion of palm print, fingerprint and face. The matching score level is carried out for fusion of three biometric traits based on the proximity of feature vector and template.Jian yang et al., [11] proposed sparse representation classifiers steered discriminative projection with application to face recognition. Sparse representation based classifier $(\mathrm{SRC})$ was used to direct the design of a dimensionality reduction method SRC - DP, in which the SRC achieved better performance and become more efficient. Meng yang et al., [12] proposed robust Kernel representation model with statistical local feature is used for face identification for different conditions, including variations of illumination, expression misalignment and pose. Javier Galbally et al., [13] proposed multiple biometric systems to detect different types of fraudulent access attempts using software based fake detection method. AarohiVova et al., [14] proposed Support Vector Machine (SVM) based fusion of match scores are used for face and fingerprintbiometric traits. The statistical analysis of different kernel methods is used for training SVM. The polynomial kernel radial basis function kernel and multiplier perceptron kernel are used for face and fingerprint identification. Dinakardas et al., [15] proposed principal component analysis is used to extract the features of the fingerprint and iris images.For face image fisher faces are used. The method of minutiae extraction for fingerprint and LBF feature for iris image is used. Jossy P. George et al., [16] proposed transforms domain fingerprint identification based on DTCWT. The test image features are compared with database images using Euclidean distance. 


\section{PROPOSED MODEL:}

In this section, the new concepts of convolution of original face image with DWT of original face image along with compression of database feature vectors are introduced. The block diagram of proposed model is shown in Figure 1.

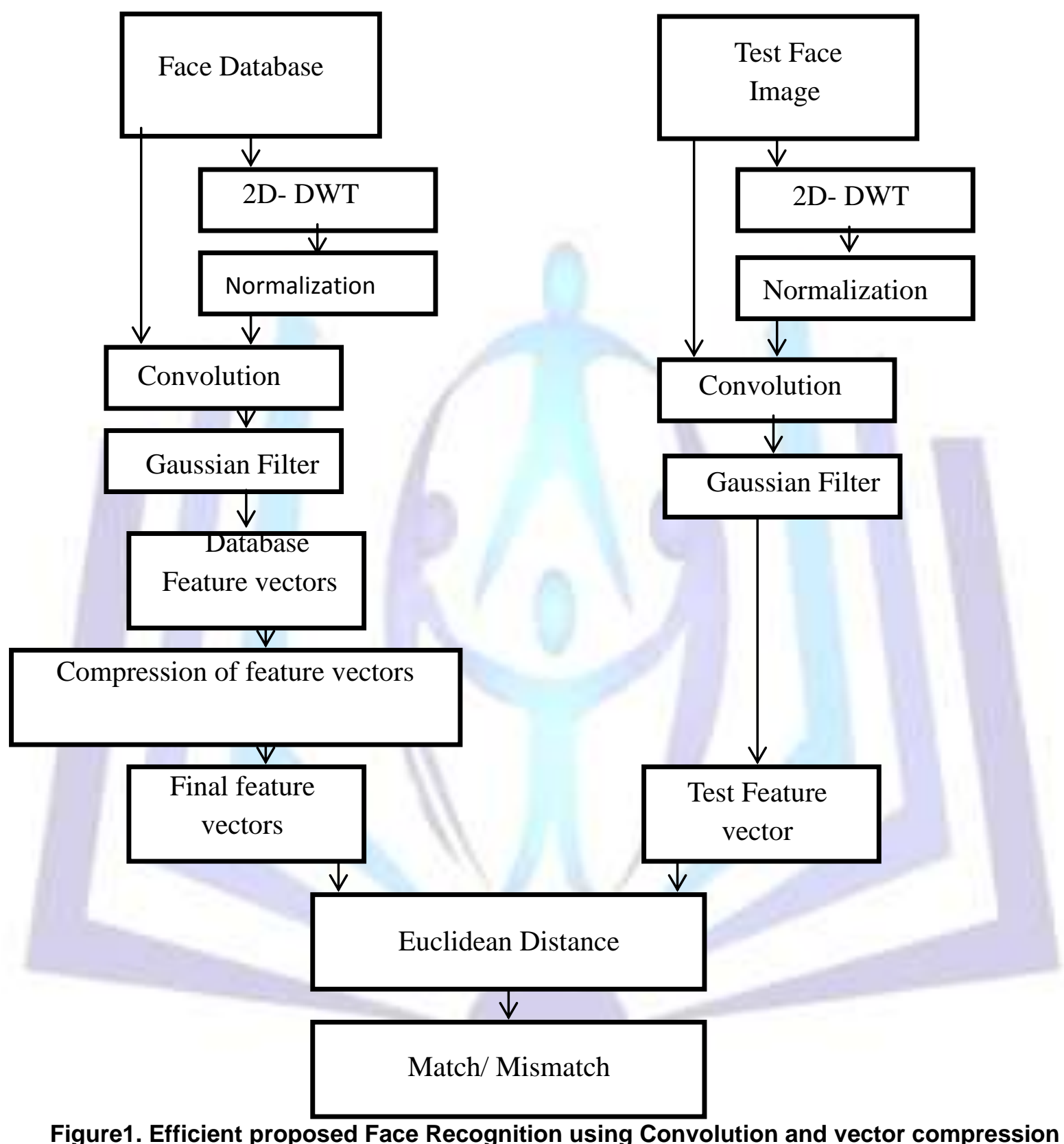

\subsection{Face Database}

The standard face databases such as ORL, JAFFE, Yaleand Indian male and Indian female are considered to test the proposed model.

3.1.1 JAFFE Database [17]:The face database has ten persons with twenty image samples per person. This image samples of one person are shown in fig 2 with facial expressions biz., angry, happy, neutral, fear, surprise etc., this eight persons with seven samples per person are considered to a creates database to compute FRR and TSR. This remaining five persons of the total database are considered to compute FAR. 

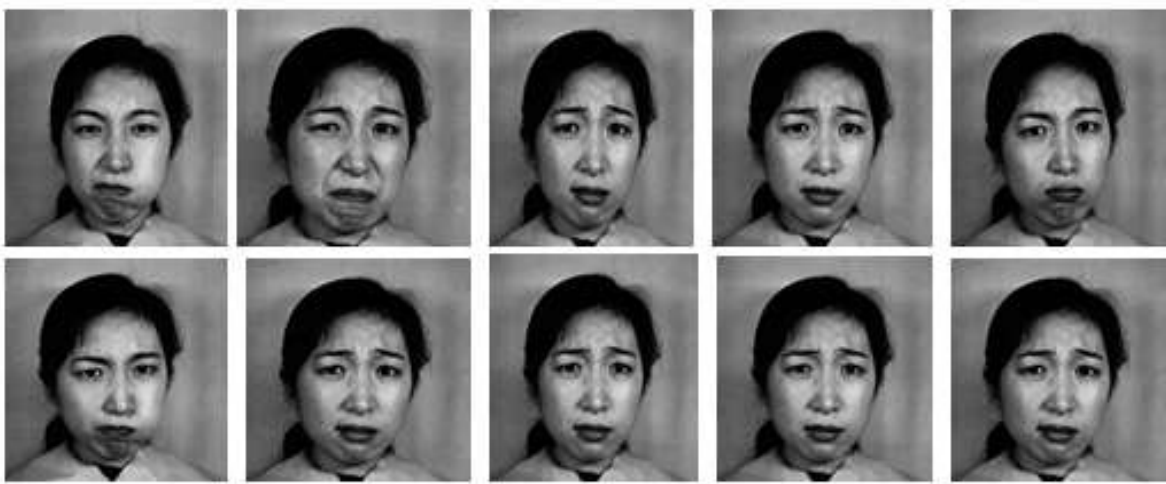

Fig 2: Samples of JAFFE face images of person

3.1.2 ORL Database [18]:This database has forty persons with ten samples per persons these surface samples of a person are captured as different times by varying lighting intensity, facial expressions and facial objects. The performance parameters such as FRR, TSR are compared by considering forty persons with six samples per persons in the database. The parameter FAR is compared by considering remaining ten persons with one sample per persons in the test image section ten image samples of the database are shown in the fig 3.
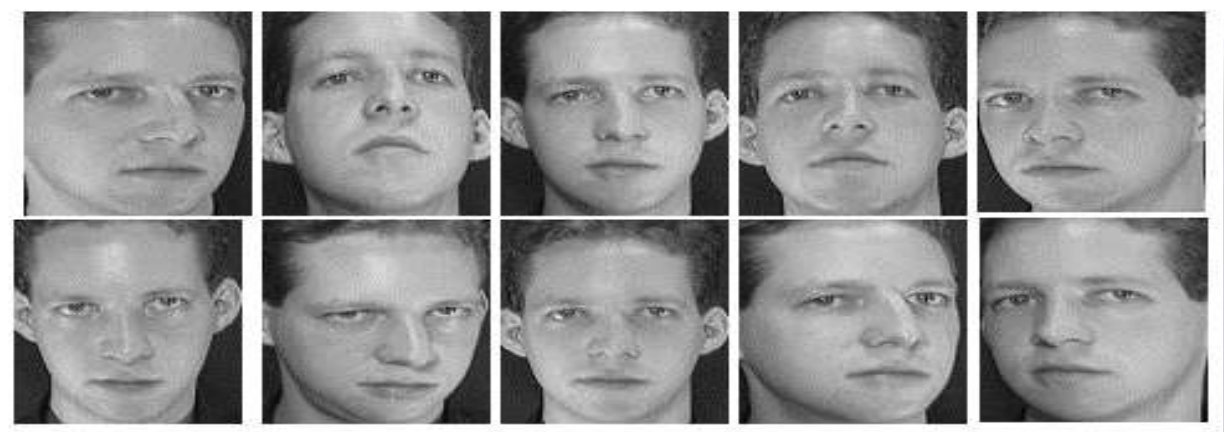

Fig 3: Samples of ORL face images of person

3.1.3 Yale face database [19]:The fifteen persons with eleven image samples per persons i.e., 165 image samples in total are present in the database. The number of image samples of a person has facial expressions and occlusion of eyes with eye glasses. The size of each face image is $243 \times 320$ with gray levels.

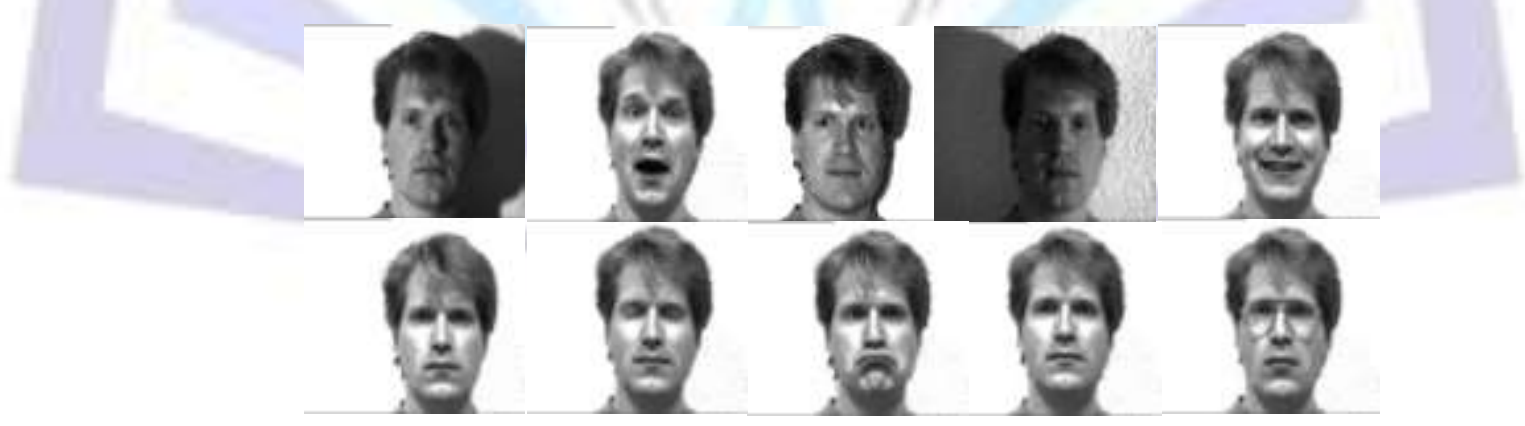

Fig 4: Samples of Yale face images of person

3.1.4 Indian male[20]:The database has twenty persons with eleven samples per persons the images work captured with frontal position variations. The number of samples of a single person with frontal orientations is shown in figure 5. The twelve persons with six samples per persons are considering creating database to compute performance parameters FRR and TSR. The remaining eight persons with one samples per persons are consider in the test section to compute FAR. 

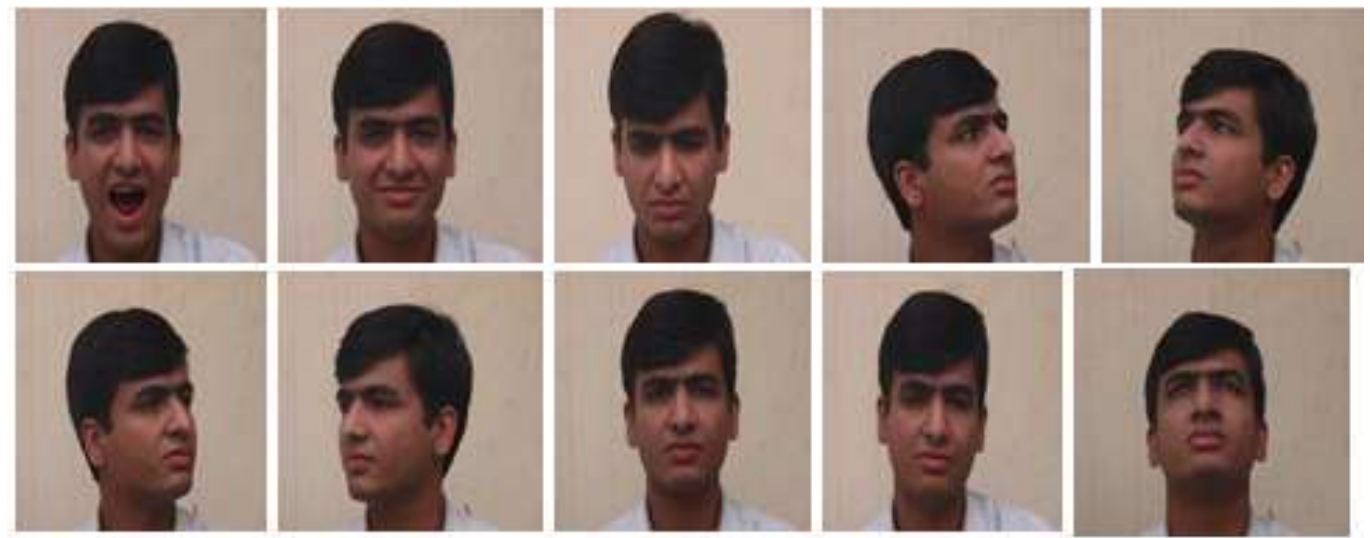

Fig 5: Samples of Indian male data base face images

3.1.5 Indian female[20]:The database has twenty two female persons with eleven samples per person. The each image of a person has variations in pose and expression as shown in figure 6 . The fifteen persons with seven image samples per persons are considered to find FRR and TSR parameters. The remaining seven persons with one sample per person are considered in the test section to compute FAR performance parameters.
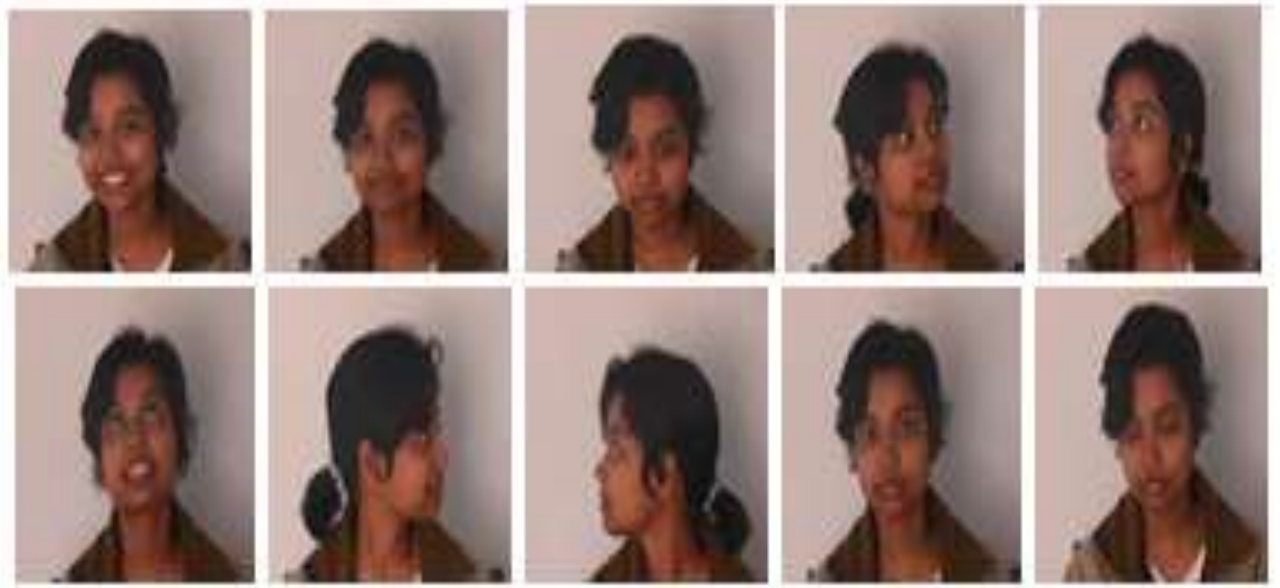

Fig 6: Samples of Indian female database face images

3.1.1 D-Discrete Wavelet Transform [21]:The transformation is used in an image processing to remove noise effectively and also compress an image. The filter bank combinations of low pass and high pass filters are used on rows and columns of an image to derive one approximation band and three detailed bands. The approximation band is an output of low pass filter and has significant information of an image. The detailed bands are outputs of high pass and combinations of high pass-low pass filters and have detailed information such as diagonal, horizontal and vertical information of an image. The DWT decomposes an image into four sub bands such as approximation band, vertical band, horizontal band and diagonal band in each level. The maximum number of decomposition levels equal to $\log _{2} N$ for $\mathrm{N} \times \mathrm{N}$ image size. The rows of images are passing through low pass and high pass filter to generate corresponding low and high frequency coefficients. The columns of an image are passing through low pass and high pass filter along with rows of low pass filter to generate approximation and vertical bands. The columns again passed through low pass and high pass filter along with output of row high pass filter to generate horizontal and diagonal bands. The one level DWT decomposition on an image using filter are shown in fig 7 and the corresponding image representation is shown in fig 8. 


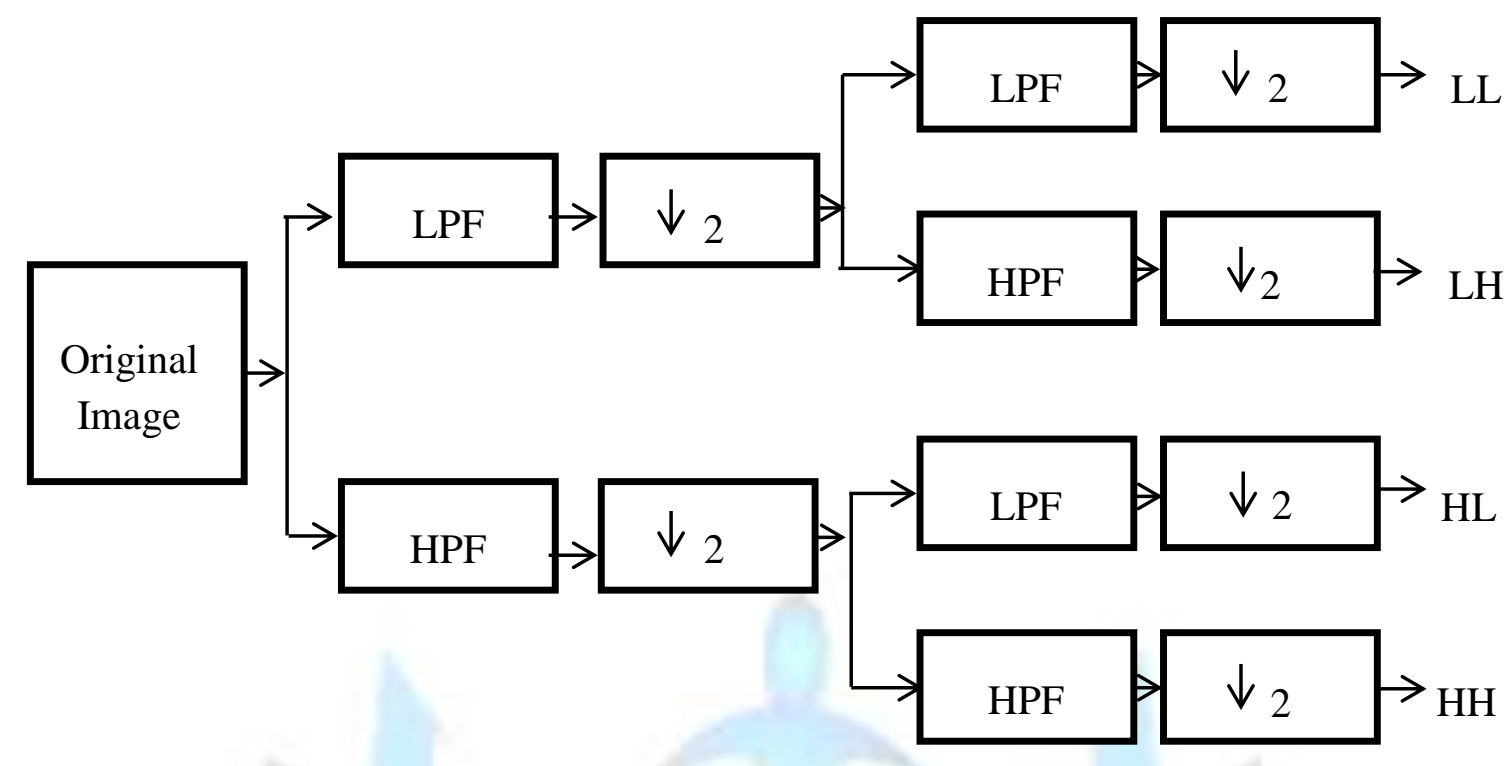

Fig7: The One level Decomposition of DWT using Filter

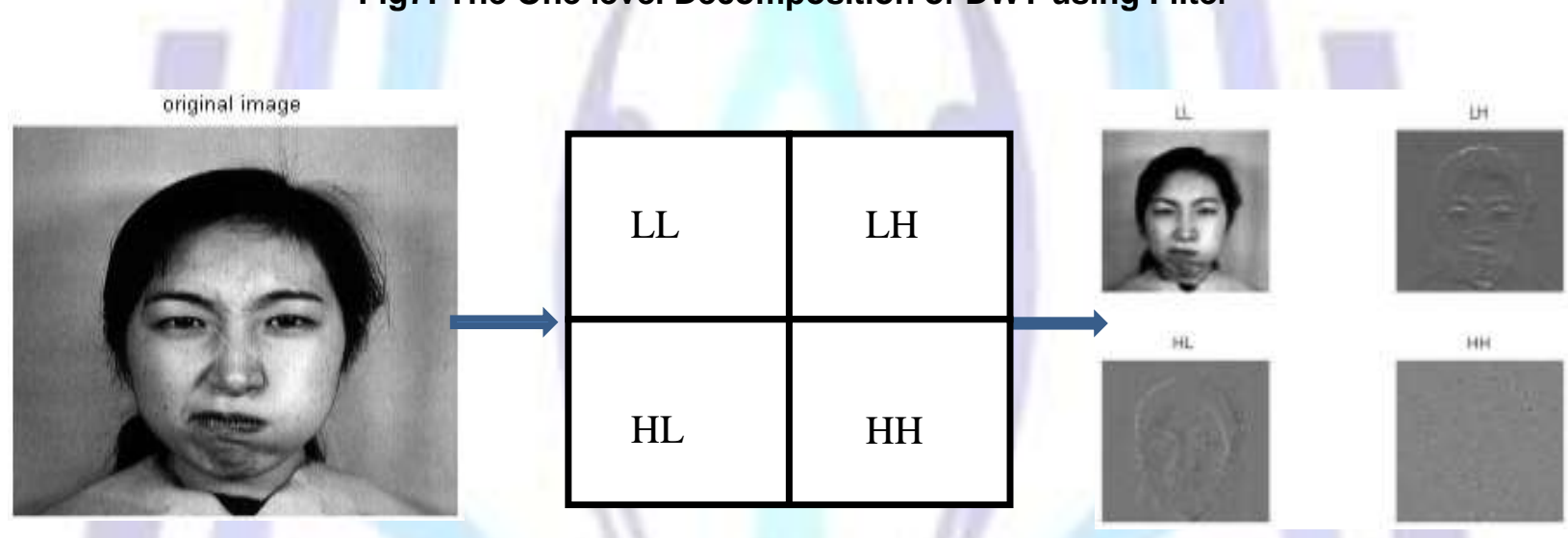

Fig 8: DWT decomposition of an image

The LL band has significant information of an original image. The $\mathrm{LH}, \mathrm{HL}$ and $\mathrm{HH}$ bands has vertical, horizontal and diagonal information of an original image. The original image can be reconstructed by considering only LL band image and omitting other insignificant information from other bands. The DWT is applied on face databases such as JAFEE, ORL, and Yale, Indian male and Indian female of resized dimensions of $256 \times 256$. The LL band is considered for further processing as it has significant information of face images and has less noise component.

\subsection{Normalization:}

Normalization used in the proposed method is to convert high values of LL band coefficients into a range of moderate values. The normalization is applied on LL sub-band coefficients of an image to convert high coefficients values into lower values. Each LL coefficientvalues are divided by maximum coefficient value to convert $L L$ coefficient values range from 0 to 1 , as given in equation (1).

$$
\text { New LL coefficient values }=\frac{\text { old coefficient values }}{\text { maximum value of LL coefficient }}
$$

The advantage of normalization is the number of bits required to represent each LL coefficient reduces from more than eight bits to less than eight bits. The real time system complexity and memory reduces, whereas speed of computation increases.

\subsection{Convolution:}

The original face image is convolved with LL sub-band of original face image. The 2D-Convolution is used for smoothing, sharpening and edge detection of an image effectively. In the proposed method, the DWT is applied on the original face image of size $256 \times 256$ to produce four sub-bands, say LL, LH, HL and HH. The approximation LL Sub-band of size 128 $x 128$ is convolved with original spatial domain face image of size $256 \times 256$ to obtain modified original face object. The 
coefficients of the modified objects classify similar and dissimilar images effectively. The 2D-Convolultion of two images $x$ $(m, n)$ and $h(x, y)$ is given in equation(2).

$$
\mathrm{X}(\mathrm{m}, \mathrm{n}){ }^{*} \mathrm{~h}(\mathrm{~m}, \mathrm{n})=\sum_{i=0}^{w-1} \quad \sum_{j=0}^{H-1} \boldsymbol{x}(\boldsymbol{i}, \boldsymbol{j}) \boldsymbol{h}(\boldsymbol{m}-\boldsymbol{i}, \boldsymbol{n}-\boldsymbol{j})
$$

Where $\mathrm{w}$ and $\mathrm{H}$ are the width and height of an image. The resultant output of convolution matrix of size $(\mathrm{w}+\mathrm{H}-1){ }^{*}(\mathrm{w}+\mathrm{H}-1)$. The example of $2 \mathrm{D}$-convolution is explained by considering original matrix of size $4{ }^{\star} 4$ and $\mathrm{LL}$ band of original matrix of size $2^{*} 2$ is as follows:

$$
\text { Original matrix }=\left[\begin{array}{cccc}
126 & 98 & 94 & 100 \\
82 & 77 & 96 & 98 \\
80 & 104 & 146 & 129 \\
79 & 78 & 118 & 134
\end{array}\right]
$$

$$
\text { LL band of original matrix }=\left[\begin{array}{cc}
191.5000 & 214.000 \\
170.5000 & 263.5000
\end{array}\right]
$$

$$
\text { Normalized matrix }=\left[\begin{array}{cc}
0.7268 & 0.8121 \\
0.6471 & 1
\end{array}\right]
$$

Original matrix $4 * 4$ is convolved with $2 * 2$ Normalized matrix to get $5 \times 5$ output convolution matrix given in equation( 6 ).

Convolution output matrix $=\left[\begin{array}{ccccc}91.5712 & 173.5522 & 147.9051 & 118.0873 & 113.7002 \\ 141.1233 & 311.9677 & 291.1271 & 333.7761 & 219.5901 \\ 111.1992 & 272.3776 & 329.6869 & 371.7362 & 202.7666 \\ 109.1784 & 268.1404 & 347.5750 & 422.6888 & 237.8273 \\ 51.1176 & 129.4706 & 154.3529 & 204.7059 & 134\end{array}\right]$

The linear convolution matrix has unique coefficient values compared to original matrix coefficient values; hence the concept of convolution is used for better classification of images.

\subsubsection{Gaussian Filter:}

It is applied on output of convolution block to enhance further the quality of input face images by removing high frequency edges and helps in improving the matching accuracy.An example of Gaussian filter on convolution output matrix is as follows. The Gaussian filter mask matrix is applied on convolution output matrix is given in matrices 7 and 8 .

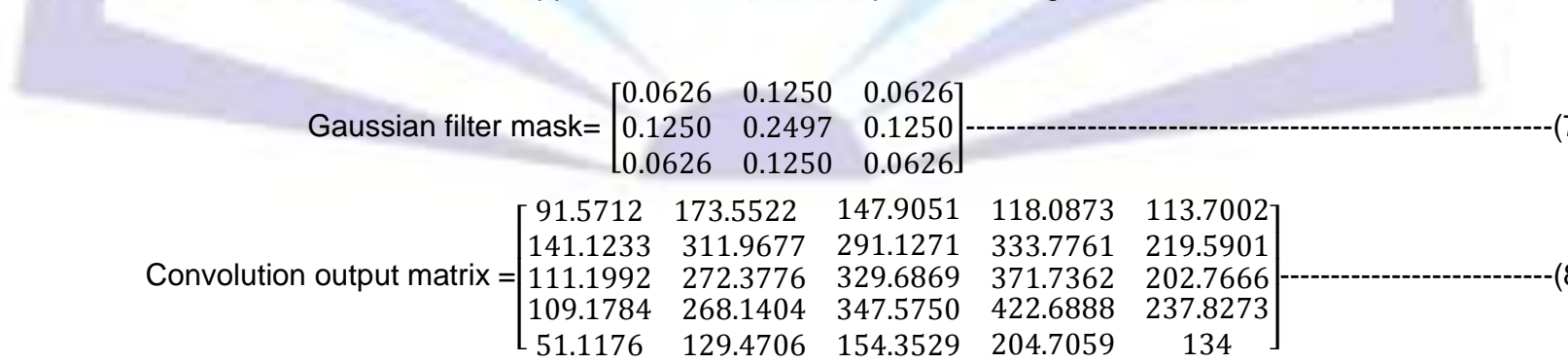

The procedure for Gaussian filter output is as follows.

1. The Gaussian filter mask $(\mathrm{h}(\mathrm{x}, \mathrm{y}))$ is generated using Gaussian mask coordinators and standard deviation as given in matrix 9 and equation 10 .

$$
\text { Gaussian mask coordinators }=\left[\begin{array}{ccc}
(-1,-1), & (-1,0), & (-1,1) \\
(0,-1), & (0,0), & (0,1) \\
(1,-1), & (1,0), & (1,1)
\end{array}\right]
$$




$$
h_{g}(x, y)=e^{\frac{-\left(x^{2}+y^{2}\right)}{2 \sigma^{2}}}
$$

Where, $\sigma_{=}$standard deviation $=0.85$

$h_{g}(-1,-1)=e^{\frac{\left.-(-1)^{2}+(-1)^{2}\right)}{2(0.85)^{2}}}=0.25055344$

$h_{g}(0,-1)=e^{\frac{-(0)^{2}+(-1)^{2}}{2(0.85)^{2}}}=0.500553134$

Similarly other coordinate coefficient values are computed and are given in matrix 11 .

$$
h_{g}(x, y)==\left[\begin{array}{ccc}
0.25055344 & 0.50055314 & 0.25055344 \\
0.50055314 & 1 & 0.50055314 \\
0.25055344 & 0.50055314 & 0.0626
\end{array}\right]
$$

Gaussian filter mask coefficient are computed using equation 12 and in matrix 13.

$$
\text { Filter mask }=\mathrm{h}(\mathrm{x}, \mathrm{y})=\frac{h_{g}(x, y)}{\sum_{x} \sum_{y} h_{g}}
$$

Where $\sum_{x} \sum_{y} h_{g}=4.004426296$

$$
H(x, y)=\left[\begin{array}{lll}
0.0626 & 0.1250 & 0.0626 \\
0.1250 & 0.2497 & 0.1250 \\
0.0626 & 0.1250 & 0.0626
\end{array}\right]
$$

2.Append Zeros to the surroundings of convolution output matrix.

$\mathrm{X}=\left[\begin{array}{ccccccc}0 & 0 & 0 & 0 & 0 & 0 & 0 \\ 0 & 91.57 & 173.55 & 147.90 & 118.08 & 113.70 & 0 \\ 0 & 141.12 & 311.96 & 291.12 & 333.77 & 219.59 & 0 \\ 0 & 111.19 & 272.37 & 329.68 & 371.73 & 202.76 & 0 \\ 0 & 109.17 & 268.14 & 347.57 & 422.68 & 237.82 & 0 \\ 0 & 51.11 & 129.47 & 154.35 & 204.70 & 134 & 0 \\ 0 & 0 & 0 & 0 & 0 & 0 & 0\end{array}\right]$

Place Gaussian filter mask of size $3^{*} 3$ generated in step 1 on top left portion of $X$ and multiply element by element.

$$
\begin{aligned}
& \mathrm{X}_{1}=\left[\begin{array}{ccc}
0 & 0 & 0 \\
0 & 91.57 & 173.55 \\
0 & 141.12 & 311.96
\end{array}\right] *\left[\begin{array}{ccc}
0.0626 & 0.1250 & 0.0626 \\
0.1250 & 0.2497 & 0.1250 \\
0.0626 & 0.1250 & 0.0626
\end{array}\right] \\
& \mathrm{X}_{1}=\left[\begin{array}{ccc}
0 & 0 & 0 \\
0 & 22.86 & 21.69 \\
0 & 17.64 & 19.52
\end{array}\right]
\end{aligned}
$$

$$
\sum X_{1}=81.7215
$$

The $3 \times 3$ Gaussian mask is shifted right and down on $X$ to compute other coefficient value of Gaussian filter output given in matrix 17.

The output of Gaussian filter $=\left[\begin{array}{ccccc}81.7215 & 139.3161 & 157.6848 & 150.8504 & 98.9874 \\ 127.4855 & 230.2479 & 275.4217 & 265.6029 & 170.5192 \\ 129.4008 & 251.2676 & 326.3108 & 322.5293 & 201.6111 \\ 106.2149 & 214.7281 & 294.8670 & 302.1430 & 190.3905 \\ 59.3737 & 120.1119 & 166.9891 & 176.6282 & 115.2269\end{array}\right]$ 
The 2-D matrix is converted into single column vector.

\subsection{Compression of feature vectors:}

The more number of sample images of single person are converted into single sample per person using compression thesix column vector features of sixface images of single person, say 1_1, 2_1, 3_1, 4_1, 5_1 and 6_1, are converted into single feature column vector by taking average of six column features as shown in fig 9 . The advantages of converting six columns into one column are (i) Time to compare test image with database images reduces (ii) Memory requirement in real time system reduces (iii) The features of single column are more effective compared to six column vector features.
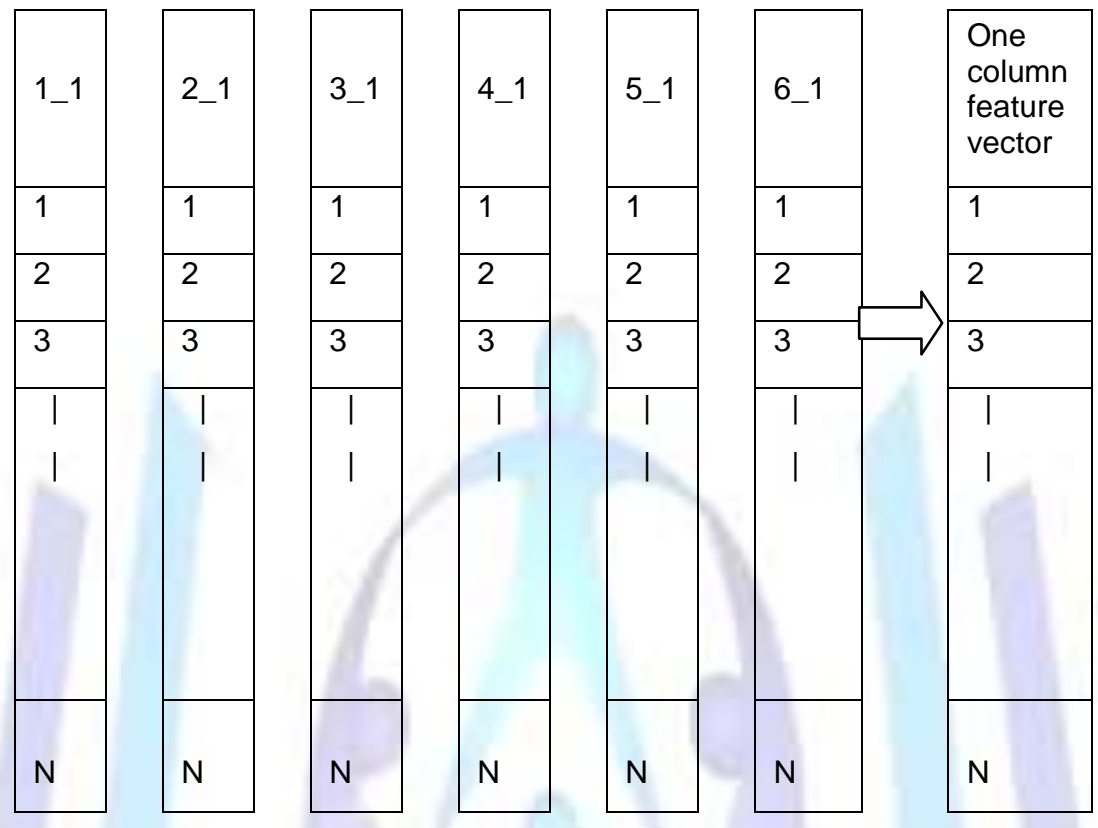

Fig 9: Compression of column vectors

3.6 Test Image Features: The features are extracted from face image similar to that of database face images to form single column feature vector.

\subsection{Euclidean distance:}

The distance between database features $\left(p_{i}\right)$ and test features $\left(q_{i}\right)$ is given in equation (18).

$$
\mathrm{ED}=\sqrt{\sum_{i=1}^{N}\left(p_{i}-q_{i}\right)^{2}}
$$

Where, $\mathrm{N}=\mathrm{No}$ of coefficients in a vector.

$\mathrm{Pi}=$ Coefficient values of vectors in the database.

$\mathrm{Qi}=$ Coefficient values of vectors in the test image.

The ED is used to find similarities and dissimilarities among face images to test performance of biometric system.

\section{PERFORMANCE ANALYSIS OF PROPOSED METHOD:}

In this section, the definitions of performance parameters and experimental results of various techniques are discussed.

\subsection{Definition of Performance Parameters:}

4.1.1 False Rejection Rate (FRR):It is defined as the probability of genuine person being rejected as an imposter. It is the ratio of number of genuine persons rejected to the total number of persons inside the database and is computed in equation (19).

$$
\mathrm{FRR}=\frac{\text { number of genuine person rejected }}{\text { total number of persons inside the database }}
$$

4.1.2 False Acceptance Rate (FAR):Is defined as the probability of imposter being accepted as genuine persons. It is ratio of imposters accepted as genuine persons from outside the database to the total number of persons in the outside database and is computed in equation (20). 
$\mathrm{FAR}=\frac{\text { number of imposter accepted as genuine person }}{\text { totalnumber ofpersons in the outside database }}$

4.1.3 Equal Error Rate (ERR):Is define as error tradeoff between FRR and FAR i.e., FAR = FRR for a particular threshold value. A lower EER value indicates better performance of biometric systems.

4.1.4 Total Success Rate (TSR):Is the measure of accuracy of biometric systems. It is the ratio of the total number of genuine persons identified correctly in the data base to the total number of persons inside the database and is computed in equation (21).

$$
\mathrm{TSR}=\frac{\text { genuine persons identified correctly }}{\text { total number ofpersons inside the database }}
$$

4.1.5 Optimum Total Success Rate (Opt.TSR):The value of TSR corresponding to the EER for specified threshold.

4.1.6 Maximum Total Success Rate (Max. TSR):The value of maximum TSR for specified threshold value irrespective of error values.

\subsection{Performance Analysis:}

The performance parameters such as FRR, FAR, TSR, EER and Max. TSR are computed and compared for different face databases andtechniques to verify the efficiency of biometric system.

4.2.1 Analysis using JAFEE database:The database is created by considering seven persons with six samples per person i.e. forty two samples inside the database.

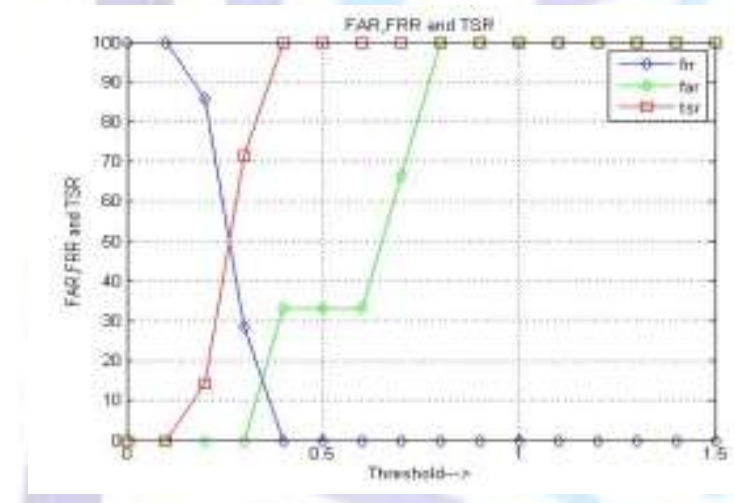

(a) DWT

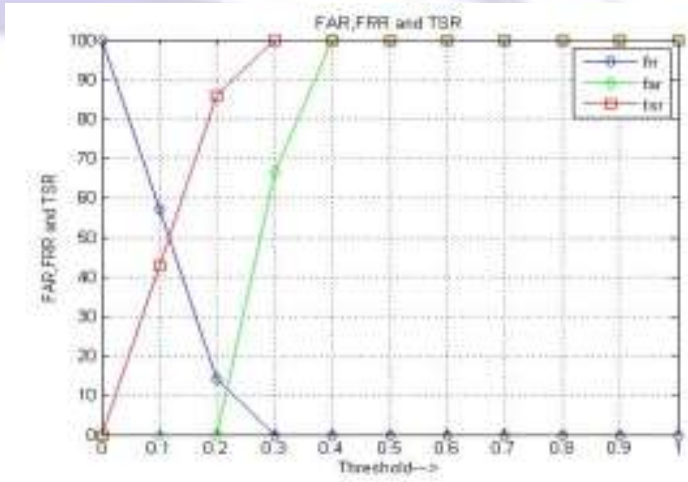

(c) Convolution

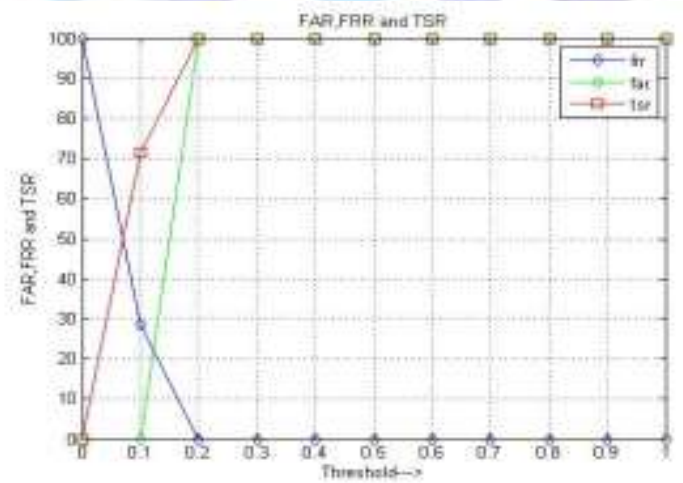

(b) FFT

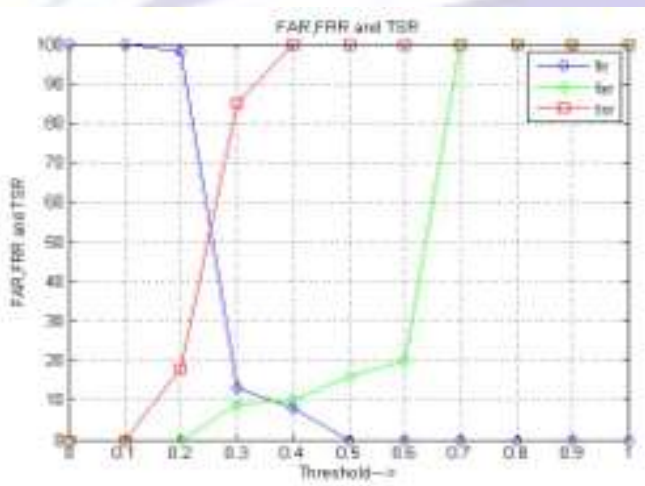

(d) Proposed method

Figure 10.FAR, FRR and TSR vs. Threshold for JAFEE Database

The ninth image of every person is considered as test face image. Three persons with one samples per persons are considered as outside database The inside and outside databases are used to compute errors such as FRR and FAR 
respectively. The variations of FAR, FRR and TSR with respect to threshold are shown in figure 10. For DWT, FFT, convolution and proposed method. The values of FRR are decreased as threshold values increases. The values of FAR and TSR increases with increase in threshold values. The ERR values for DWT, FFT, convolution and proposed method are 15, 22, 11 and 10 respectively. The optimum TSR values for DWT, FFT, convolution and proposed method are75, 76, 90 and 95 respectively. The maximum TSR values for DWT, FFT, convolution and proposed method are 100 . It is observed that the value of EER is less and optimum TSR value is high in the case of proposed method compared to DWT, FFT and convolution techniques.

\subsubsection{Analysis using ORL face database:}

The database is created by considering 30 persons with 6 samples per person i.e., 180 samples inside the database and the ninth image of every person is considered as test face image. Ten persons are considered as outside database with one sample per person. The inside and outside databases are used to compute errors such as FRR and FAR respectively. The variations of FAR, FRR and TSR with respect to threshold are shown in figure 11, for DWT, FFT, convolution and proposed method, the values of FRR are decreases as threshold values increases. The values of FAR, TSR increases with increase in threshold values. The EER values for DWT, FFT, convolution and proposed method are 30, 36, 28 and 18 respectively. The optimum TSR values for DWT, FFT, convolution and proposed method are 62, 60, 70 and 73 respectively. The maximum TSR values for DWT, FFT, convolution and proposed method are 90, 83.33, 93.33 and 93.33 respectively.It is observed that the value of EER is less and optimum TSR value is high in the case of proposed methodcompared to DWT, FFT and convolution techniques.

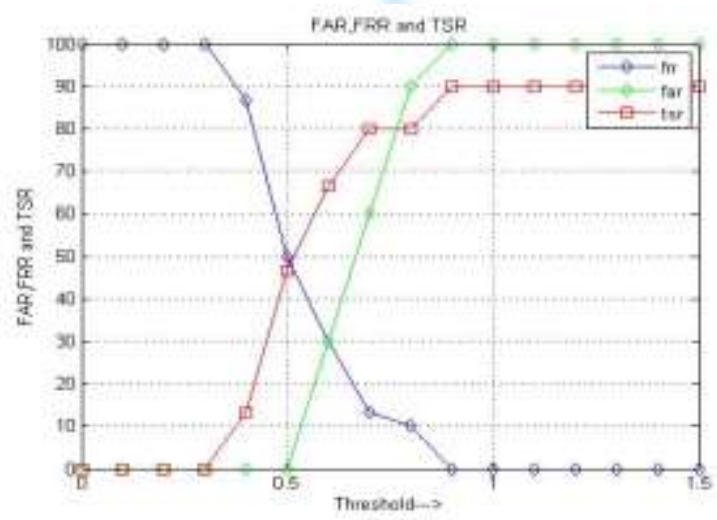

(a) DWT

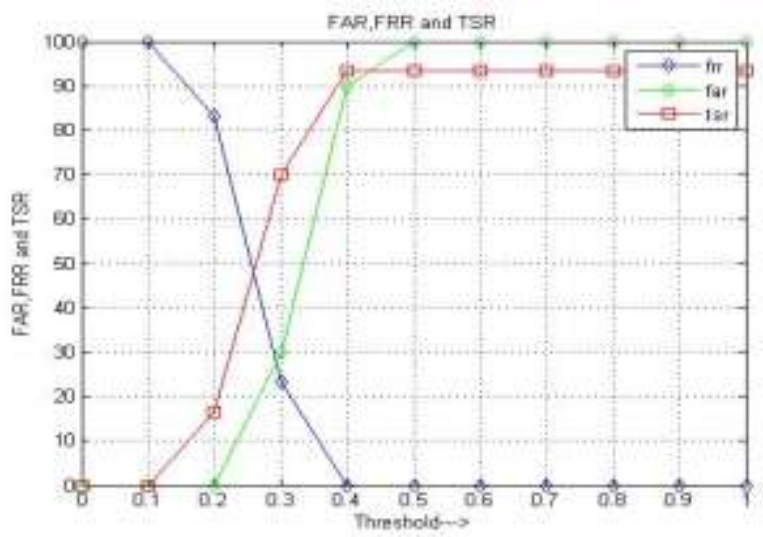

(c) Convolution

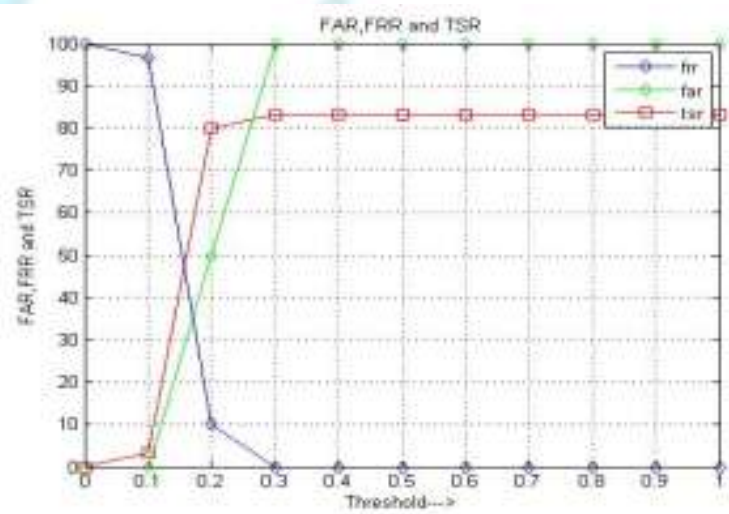

(b) FFT

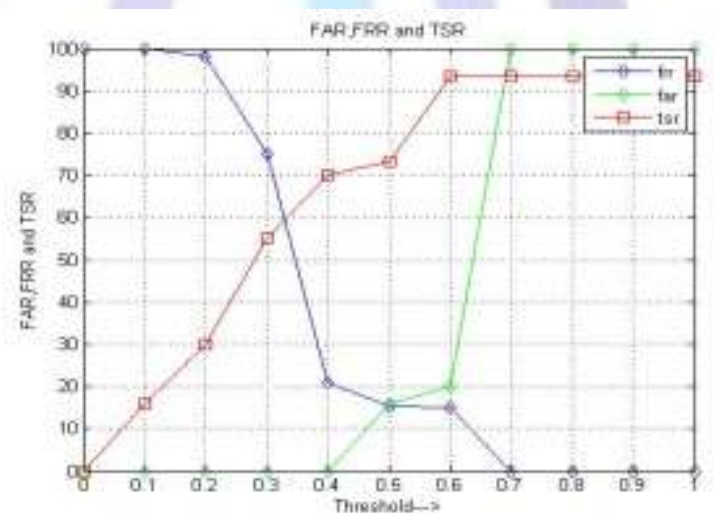

(d) proposed method

Figure 11. FAR, FRR and TSR vs. Threshold for ORL Database

4.2.3 Analysis using Yale face database:The database is created by considering 11 persons with 6 samples per person i.e., 60 samples inside the database and the ninth image of every person is considered as test face image. 


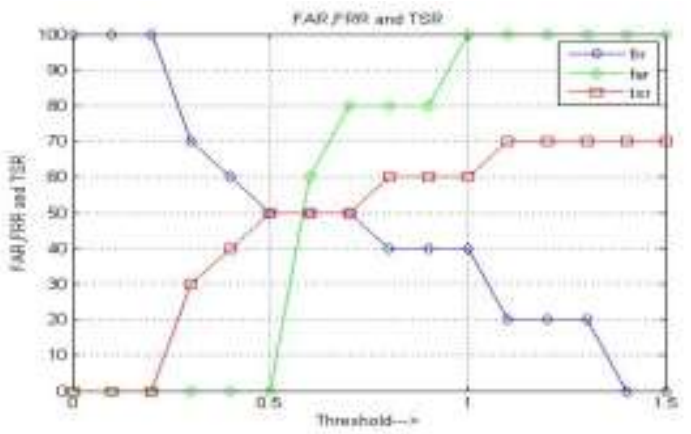

(a) DWT

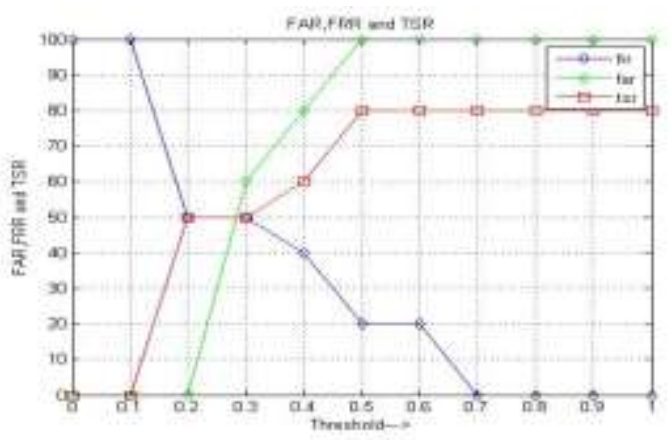

(c) Convolution

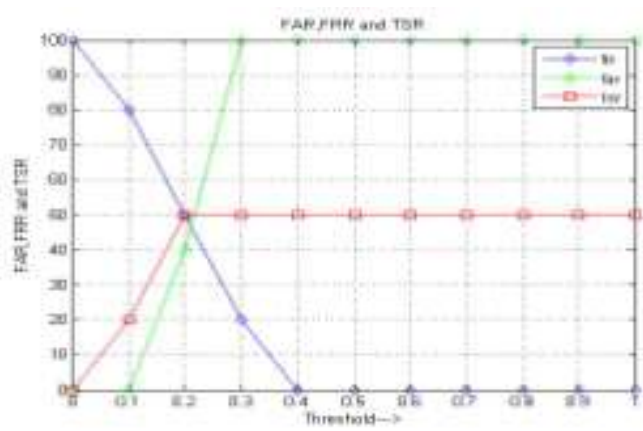

(b) FFT

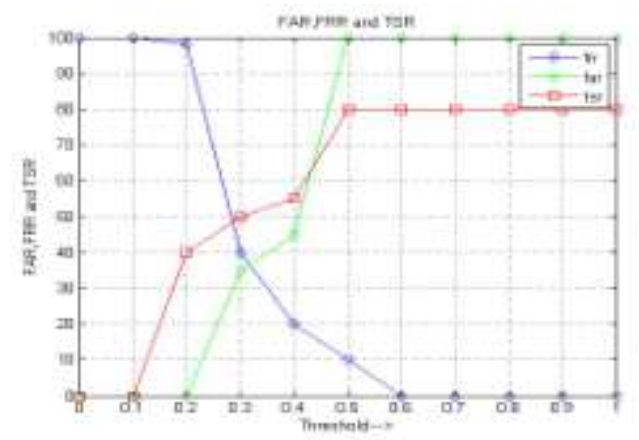

(d) Proposed method

Figure 12. FAR, FRR and TSR vs. Threshold for Yale Database

Five persons are considered as outside database with one sample per person. The inside and outside databases are used to compute errors such as FRR and FAR respectively. The variations of FAR, FRR and TSR with respect to threshold are shown in figure 12, for DWT, FFT, convolution and proposed method. The values of FRR are decreases as threshold values increases. The values of FAR, TSR increases and threshold values increase. The EER values for DWT, FFT, convolution and proposed method are 50, 48, 50 and 38 respectively. The optimum TSR values for DWT, FFT, convolution and proposed method are 50,50,50 and 51 respectively. The maximum TSR values for DWT, FFT, convolution and proposed method are 70,50, 80 and 80 respectively. It is observed that the value of EER is less and optimum TSR value is high in the case of proposed methodcompared to DWT, FFT and convolution techniques.

4.2.4 Analysis using Indian Male face database:The database is created by considering 15 persons with 6 samples per person i.e., 90 samples inside the database and the ninth image of every person is considered as test face image. Five persons are considered as outside database with one sample per person. The inside and outside databases are used to compute errors such as FRR and FAR respectively. The variations of FAR, FRR and TSR with respect to threshold are shown in figure 13, for DWT, FFT, convolution and proposed method. The values of FRR are decreases as threshold values increases. The values of FAR, TSR increases and threshold values increase. The EER values for DWT, FFT, convolution and proposed method are 57, 55, 51 and 40 respectively. The optimum TSR values for DWT, FFT, convolution and proposed method are 45, 45, 50 and 55 respectively. The maximum TSR values for DWT, FFT, convolution and proposed method are $93.33,93.33,83.33$ and 83.33 respectively. It is observed that the value of EER is less and optimum TSR value is high in the case of proposed methodcompared to DWT, FFT and convolution techniques.

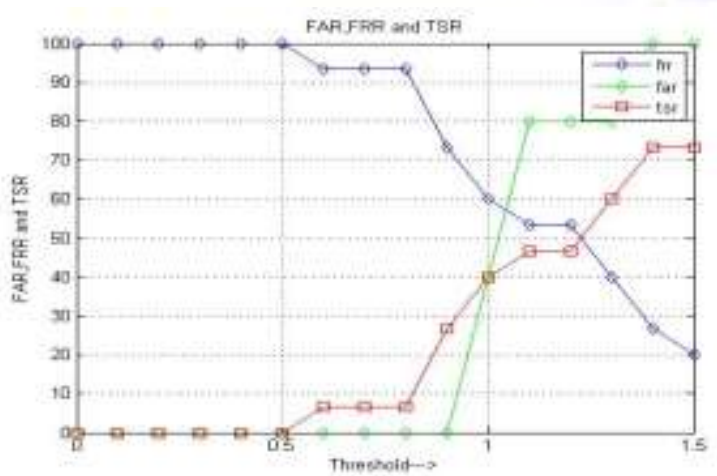

(a) DWT

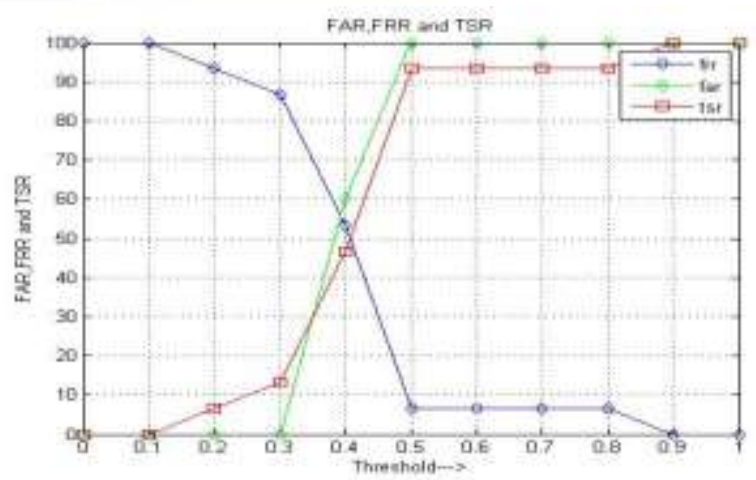

(b) FFT 


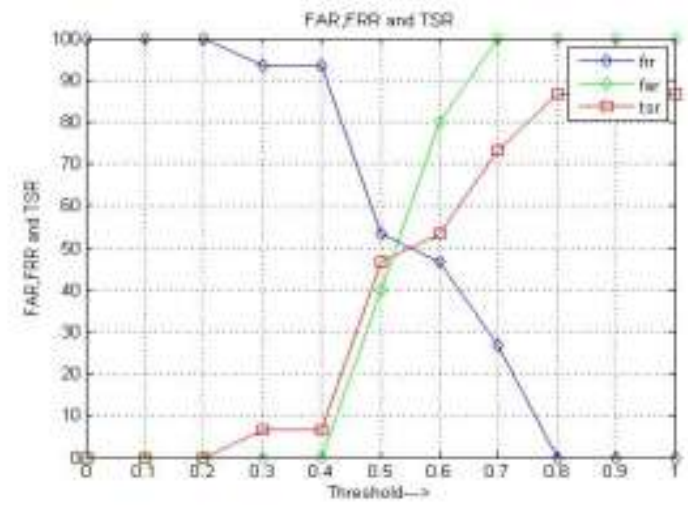

(c) Convolution

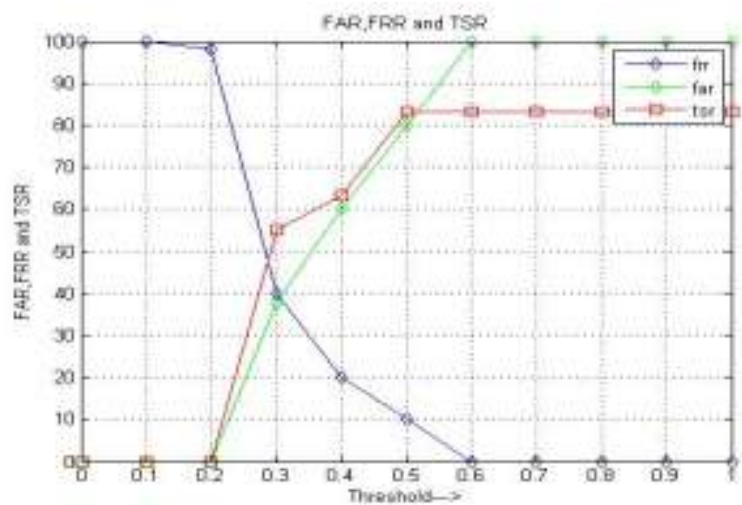

(d) proposed method

Figure 13. FAR, FRR and TSR vs. Threshold for Indian Male Database

4.2.5. Analysis using Indian Female face database: The database is created by considering 17 persons with 6 samples per person i.e., 102 samples inside the database. The ninth image of every person is considered as test face image. Five persons are considered as outside database with one sample per person. The inside and outside databases are used to compute errors such as FRR and FAR respectively. The variations of FAR, FRR and TSR with respect to threshold are shown in figure 14, for DWT, FFT, convolution and proposed method, the values of FRR are decreases as threshold values increases. The values of FAR and TSR increases with increase in threshold values. The EER values for DWT, FFT, convolution and proposed method are $28,5,0$ and 0 respectively. The optimum TSR values for DWT, FFT, convolution and proposed method are 60, 95, 93.33 and 93.33 respectively. The maximum TSR values for DWT, FFT, convolution and proposed method are 93.33. It is observed that the value of EER is less and optimum TSR value is high in the case of proposed methodcompared to DWT, FFT and convolution techniques.

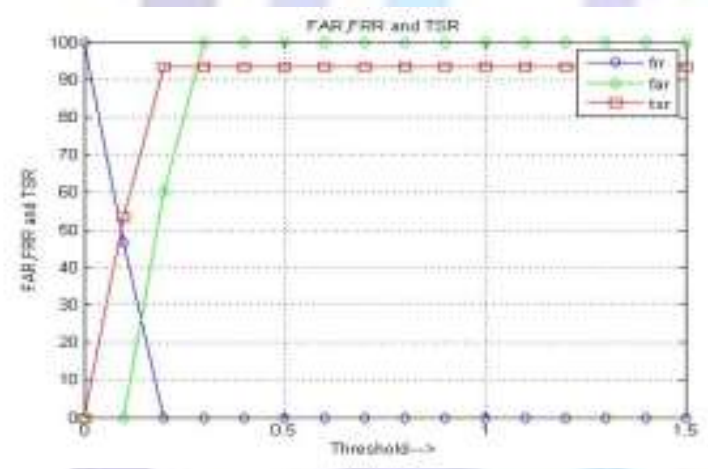

(a) DWT

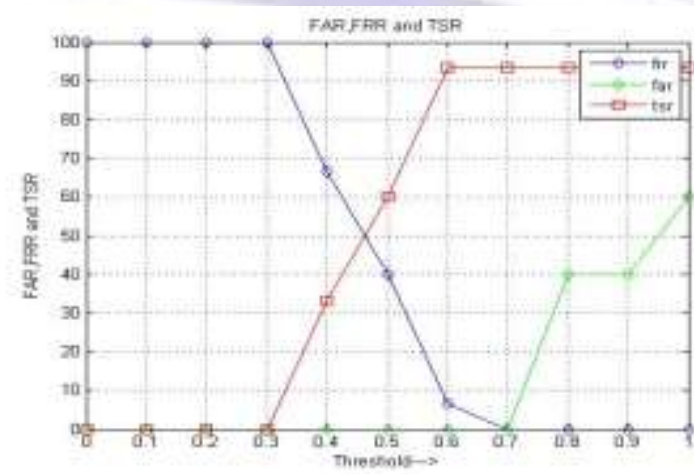

(c) Convolution

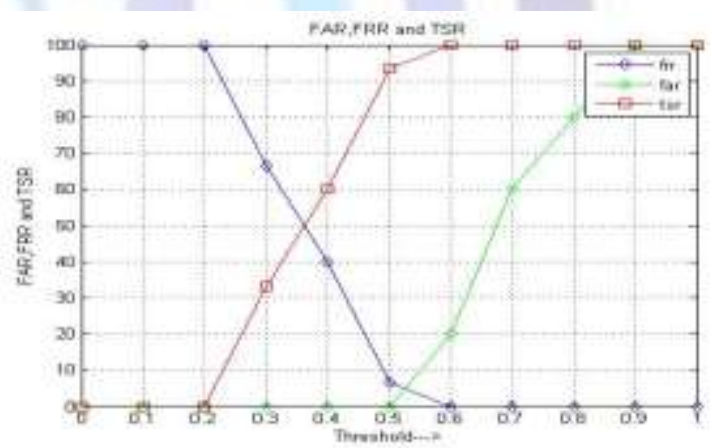

(b) FFT

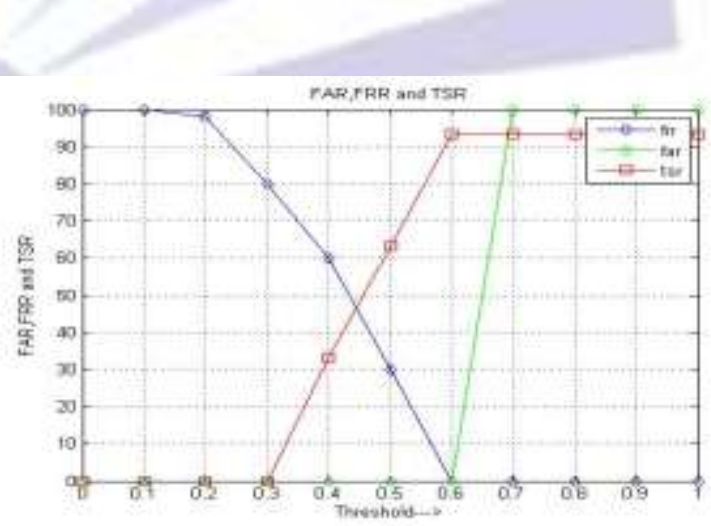

(d) Proposed method

Figure 14. FAR, FRR and TSR vs. Threshold for Indian Female Database 
4.2.6 Performance Parameter Comparisons for DWT technique:The values of EER, optimum TSR and maximum TSR are tabulated in table 1 for different face data bases using DWT technique. It is observed that the EER value is low;whereas optimum TSR and maximum TSR are high with JAFEE face data base. The Indian male face data base has high EER value and less optimum TSR value since the variations are high in the face images.

Table 1: Performance parameters for different database using DWT

\begin{tabular}{|l|l|l|l|}
\hline Databases & EER & Opt. TSR & Max.TSR \\
\hline ORL & 30 & 62 & 90 \\
\hline JAFEE & 15 & 75 & 100 \\
\hline YALE & 50 & 50 & 70 \\
\hline Indian Male & 57 & 45 & 73.33 \\
\hline Indian Female & 28 & 60 & 93.33 \\
\hline
\end{tabular}

4.2.7 Performance parameter comparison for FFT technique.The values of EER, optimum TSR and maximum TSR are tabulated in table 2 for different face databases using FFT technique. It is observed that the EER value is low, whereas optimum TSR and maximum TSR are high withIndian female face database. The Indian male face database has high EER value and less optimum TSR value since the variations are high in the face images.

Table 2: Performance parameters for different database using FFT

\begin{tabular}{|c|c|c|c|}
\hline Databases & EER & Opt. TSR & Max.TSR \\
\hline ORL & 36 & 60 & 83.33 \\
\hline JAFEE & 22 & 76 & 100 \\
\hline YALE & 48 & 50 & 50 \\
\hline Indian Male & 55 & 45 & 93.33 \\
\hline Indian Female & 5 & 95 & 100 \\
\hline
\end{tabular}

\subsubsection{Performance parameter comparison for convolution technique:}

The values of EER, optimum TSR and maximum TSR are tabulated in table 4 for different face databases using convolution technique. It is observed that the EER value is Zero, whereas high optimum TSR with Indian female face database. The Indian male face database has high EER value and less optimum TSR value since the variations are high in the face images.

Table 3: Performance parameters for different database using Convolution

\begin{tabular}{|l|l|l|l|}
\hline Databases & EER & Opt. TSR & Max.TSR \\
\hline ORL & 28 & 70 & 93.33 \\
\hline JAFEE & 11 & 90 & 100 \\
\hline YALE & 50 & 50 & 80 \\
\hline Indian Male & 51 & 50 & 83.33 \\
\hline Indian Female & 0 & 93.33 & 93.33 \\
\hline
\end{tabular}




\subsubsection{Performance parameter comparison for proposed technique.}

The values of EER, optimum TSR and maximum TSR are tabulated in table 5 for different face databases using proposed technique. It is observed that the EER value is Zero with Indian female whereas high optimum TSR and maximum TSR with Indian female face databases

Table 4: Performance parameters for different database using proposed method

\begin{tabular}{|l|l|l|l|}
\hline Databases & EER & Opt. TSR & Max.TSR \\
\hline ORL & 18 & 73 & 93.33 \\
\hline JAFEE & 10 & 95 & 100 \\
\hline YALE & 38 & 51 & 80 \\
\hline Indian Male & 40 & 55 & 83.33 \\
\hline Indian Female & 0 & 93.33 & 93.33 \\
\hline
\end{tabular}

It is observed that, the performance parameter are better in the case of proposed technique compared to DWT,FFT and convolution for all databases.

\subsubsection{Comparison of Proposed Method with existing Methods:}

The percentage TSR of proposed method for ORL database is compared with existing algorithms presented by Swarup Kumar DandpatandSukadevMeher[22],Pallavi D. Wadakar and MeghaWankhade[23], and Murugan et al.,[24] andAjay et al.,[25].is given in table 5. It is observed that the percentage TSR is high in the case of proposed method compared to existing algorithms. The performance of proposed method is better compared to existing algorithms for the following reasons. (i) Normalization performed on 2D DWT reduces the magnitude values of coefficients. ii). unique features are obtained using convolution. iii). Removal of high frequency noise components which help in matching accuracy using Gaussian filter .iv).Compression of Database features storing in to single column vector improves the matching speed.

Table 5: Comparison of TSRwith proposed and existing algorithms

\begin{tabular}{|c|c|c|c|}
\hline Sr. No & Authors & Techniques & \% TSR \\
\hline 1 & $\begin{array}{c}\text { Swarup Kumar } \\
\text { Dandpat and } \\
\text { SukadevMeher [22] }\end{array}$ & PCA+2DPCA & 90.5 \\
\hline 2 & $\begin{array}{c}\text { Pallavi D. Wadakar } \\
\text { and MeghaWankhade[23] }\end{array}$ & DWT & 90 \\
\hline 3 & Murugan et al.,[24] & Gaborfilter + & 92 \\
\hline 4 & DWT + PCA & 92.50 \\
\hline 5 & Ajay and pankaj [25] & N-PCA & 90.00 \\
\cline { 3 - 4 } & Proposed Method & PCA & 93.33 \\
\hline
\end{tabular}

\section{CONCLUSION:}

The identification of a person using physiological trait face is more efficient compared to traditional methods of recognition. In this paper feature vector compression approach for face recognition using convolution and DWT is proposed. The one level DWT is applied on face images to compress and remove noise in the face images. The LL band is considered and normalized to convert range of high coefficient values to less than one. The convolution is applied between face images and normalized LL band matrix to get better features of faces images. The Gaussian filter is applied on convolution features to enhance further quality of input images. The concept of converting many feature vectors of single person converted into single vector which reduces number of vectors and number of features of a single person. The Euclidean 
distance images to compute performance parameters. It is observed that the performance of the proposed algorithm is better compared to existing algorithm. In feature the convolution can be used in the place of Euclidean distance for the matching.

\section{References:}

[1] Ahilandswari, Prabu U, Priyadharshini G, Saranya M, Reshma Praveen N, Shanmugam M and Amudhavel, "Efficient Personal Identification using Multimodal Biometrics", IEEE International Conference on circuit, power and computing technologies,pp 46-54, 2015.

[2] Jianwuwan, Ming Yang, Yang Gao and Yinjuan Chen, "Pairwise Costs in Semi supervised Discriminant Analysis for Face Recognition", IEEE Transactions on Information Forensics and Security, vol.9, No.10, pp 1569-1580, October, 2014.

[3] Hailing Zhou, AjmalMian, Lei Wei, Doug Creighton, Mo Hossny and SaeidNahavandi, "Recent Advances on Singlemodal and Multimodal Face Recognition", IEEE Transaction on Human Machine Systems, vol.44, No.6, pp 701-716, December, 2014.

[4] Rupali L. Telgad, P.D. Deshmukh, MGM's, G.Y.P.C.C.S, I.T. Aurangabad and Almas M.N. Siddiqui, "Combination Approach to Score Level Fusion for Multimodal Biometric System by using Face and Finger Print", IEEE International Conference on Recent Advances and Innovations in Engineering, pp1-8, May, 2014.

[5] Madeena Sultana, Marina Gavrilova, Redaathajj and Svetlana Yanushkevich, "Adaptive Multi-Stream Score Fusion for Illumination Invariant Face Recognition" IEEE conference on Computational Intelligence in Biometrics and Identity Management, pp 94-101, 2014.

[6] Jiven Lu, Gang Wann and Pierre Maulin, "Localized Multi-Feature Metric Learning for Images Set Based Face Recognition" IEEE Transaction on Circuits and Systems for Video Technology,Issue 99,pp. 1 - 13, 2015.

[7] Faisal Ahmed, EmamHossain, A.S.M. Hossain Bari and ASM Shihavuddin "Compound Local Binary Pattern (CLBP) for Robust Facial Expression Recognition" IEEE International Symposium on Computational Intelligence and Informatics, pp.391-395,2011.

[8] XuXiaona, Pan Xiuqin, Zhao Yue and PuQiumei, "Research on Kernel-Based Feature Fusion Algorithm in Multimodal Recognition" IEEEInternational Conference on Information Technology and Computer Science,vol 2,pp. $3-6,2009$.

[9] Susma S Patil, Gajendra Singh Chandel and Ravindra Gupta, "Fingerprint Image Enhancement Techniques and Performance Evaluation of the SDG and FFT Fingerprint Enhancement Techniques" International Journal of Computer Technology in Electronics Engineering, Vol 2, pp. 184 - 190, April, 2012.

[10] SheetalChaudharyandRajendraNath, "A Multimodal Biometric Recognition System Based on Fusion of Palmprint, Fingerprint and Face", IEEEInternational Conference on Advance in Recent Technologies in Communication and Computing, pp $596-600,2009$.

[11] JainYang, Delin Chu, Lei Zhang, Yong Xu and Jingyu Yang, "Sparse Representation Classifier Steered Discriminative Projection with Applications to Face Recognition", IEEE Transaction on Neural Networks and Learning Systems, Vol.24, No.7, pp.1023-1035July, 2013.

[12] Mang Yang, Lei Zhang, Simon Chi-Keung Shiu and David Zhang, "Robust Kernel Representation with Statistical Local Features for Face Recognition", IEEE Transaction on Neural Networks and Learning Systems, Vol.24, No.6, pp. 900-912,July, 2013.

[13] Javier Galbally, Sebastien Marcel and Julian Fierrez, "Image Quality Assessment for Fake Biometric Detection: Application to Iris, Fingerprint and Face Recognition", IEEE Transactions on Image Processing, Vol.23, No.2, pp.710-724,Feb, 2014.

[14] AarohiVora, Chirag N Paunwala and MitaPaunwala, "Statistical Analysis of Various Kernel Parameters on SVM Based Multimodal Fusion” IEEE India Conference(INDICON), pp. 1 - 5, 2014.

[15] Dinakardas CN, S Permal Shankar and Nisha George, "A Multimodal Performance Evaluation on Two Different Models Based on Face, Fingerprint and Iris Templates", IEEE conference on Emerging trends in VLSI, Embedded system, Nano Electronics and Telecommunication system., pp. 1-6, 2013

[16] Jassy P George, Abhilash S K and Raja K B, "Transform Domain Fingerprint Identification Based on DTCWT", International Journal of Advance Computer Science and Applications, vol.3, No.1, pp 190 - 195, 2012.

[17] http://www.kasrl.org/jaffe download.html.

[18] http://www.camrol.co.uk

[19] http://vision.ucsd.edu/content/yale-face-database

[20] http://viswww.cs.umass.edu/ vidit/IndianFaceDatabase/ 
[21] ArvindBisht and Monika Gupta, "Chip Design of DWT for Image Compression", International Research Journal of Engineering and Technology, vol.2, No.4, pp 320 - 324, 2015.

[22] Swarup Kumar Dandapat and SukadevMeher, "Performance Improvement for Face Recognition using PCA and Two-Dimensional PCA," IEEE International Conference on Computer Communication and Informatics, pp. 1-5, 2013.

[23] PallaviD.Wadkar and MeghaWankhade, "Face Recognition using Discrete Wavelet Transforms," International Journal of AdvancedEngineering Technology, vol. 3,pp. 239-242, 2012.

[24] DMurugan, S Arumugam, K Rajalakshmi and Manish T, "Performance Evaluation of Face Recognition using Gabor Filter, Log Gabor filter and Discrete Wavelet Transform," International Journal of computer science and Information Technology, vol. 2, no. 1, pp. 125-132, 2010.

[25] Ajay Kumar Bansal and PankajChawla, "Performance Evaluation of Face Recognition using PCA and N-PCA", International Journal of Computer Applications, Vol. 76, No.8, pp 14-20, August, 2013.

\section{Authors}

${ }^{1}$ Ganapathi VSagar in the Dept of Electronics and Instrumentation Engineering at Dr.Ambedkar Institute of Technology, Bangalore. He obtained his B.E. degree in Instrumentation Technology from BDT, College of Davangere. His specialization in Master degree was Bio-Medical Instrumentation from VTU,Belgaum and currently he is pursuing Ph.D. in the area of Image Processing under the guidance of Dr. K Suresh Babu, Professor, Dept of Electronics and Communication Engineering, University Visvesvaraya college of Engineering, Bangalore. He has over 30 research publications in referred International Journals and Conference Proceedings. His area of interest is in the field of Signal Processing and Communication Engineering.

${ }^{2}$ Raja K.B. is a Professor, Department of Electronics and Communication Engineering, University Visvesvaraya college of Engineering, Bangalore University, Bangalore. He obtained his B.E and M.E in Electronics and Communication Engineering from University Visvesvaraya College of Engineering, Bangalore. He was awarded Ph.D. in Computer Science and Engineering from Bangalore University. He has over 180 research publications in refereed International Journals and Conference Proceedings. His research interests include Image Processing, Biometrics, VLSI Signal Processing, computer networks.

${ }^{3}$ K.Suresh Babuis a Professor, Department of Electronics and Communication Engineering, University Visvesvaraya college of Engineering, Bangalore University, Bangalore. He obtained his B.E and M.E in Electronics and Communication Engineering from University Visvesvaraya College of Engineering, Bangalore. He was awarded Ph.D. in Computer Science and Engineering from Bangalore University. He has over 40 research publications in refereed International Journals and Conference Proceedings. His research interests include Image Processing, Biometrics, VLSI Signal Processing, computer networks.

${ }^{4}$ Venugopal K.R. is currently the Principal and Dean, Faculty of Engineering, University Visvesvaraya College of Engineering, Bangalore University, Bangalore. He obtained his Bachelor of Engineering from University Visvesvaraya College of Engineering. He received his Master's degree in Computer Science and Automation from Indian Institute of Science, Bangalore. He was awarded Ph.D. in Economics from Bangalore University and Ph.D. in Computer Science from Indian Institute of Technology, Madras. He has a distinguished academic career and has degrees in Electronics, Economics, Law, Business Finance, Public Relations, Communications, Industrial Relations, Computer Science and Journalism. He has authored 27 books on Computer Science and Economics, which include Petrodollar and the World Economy, C Aptitude, Mastering C, Microprocessor Programming, Mastering C++ etc. He has
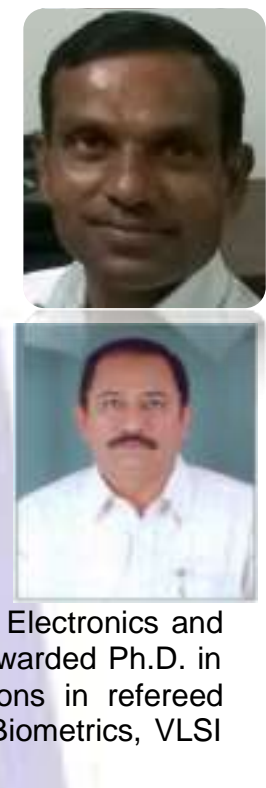
been serving as the Professor and Chairman, Department of Computer Science and Engineering, University Visvesvaraya College of Engineering, Bangalore University, Bangalore. During his three decades of service at UVCE he has over 520 research papers to his credit. His research interests include computer networks, parallel and distributed systems, digital signal processing and data mining. 